\title{
Longitudinal Relationship between Plasma Reactive Oxygen Metabolites and Periodontal Condition in the Maintenance Phase of Periodontal Treatment
}

\author{
Tatsuya Machida, ${ }^{1}$ Takaaki Tomofuji, ${ }^{1}$ Daisuke Ekuni, ${ }^{1}$ Mayu Yamane, ${ }^{1}$ Toshiki Yoneda, ${ }^{1}$ \\ Yuya Kawabata, ${ }^{1}$ Kota Kataoka, ${ }^{1}$ Naofumi Tamaki, ${ }^{2}$ and Manabu Morita ${ }^{1}$ \\ ${ }^{1}$ Departments of Preventive Dentistry, Okayama University Graduate School of Medicine, Dentistry and Pharmaceutical Sciences, \\ 2-5-1 Shikata-cho, Kita-ku, Okayama 700-8558, Japan \\ ${ }^{2}$ Department of Preventive Dentistry, Institute of Health Biosciences, The University of Tokushima Graduate School, \\ 3-18 Kuramoto-cho, Tokushima 700-8503, Japan \\ Correspondence should be addressed to Takaaki Tomofuji; tomofu@md.okayama-u.ac.jp
}

Received 3 February 2014; Accepted 4 April 2014; Published 16 April 2014

Academic Editor: Vincent Sapin

Copyright (C) 2014 Tatsuya Machida et al. This is an open access article distributed under the Creative Commons Attribution License, which permits unrestricted use, distribution, and reproduction in any medium, provided the original work is properly cited.

\begin{abstract}
Aim. The present cohort study describes the longitudinal relationship between plasma oxidative status and periodontitis progression during the maintenance phase of treatment. Materials and Methods. Forty-five patients (mean age 58.8 years) were monitored from 2008 to 2013. Periodontal conditions, including probing pocket depth (PPD) and clinical attachment level (CAL), were recorded. Measurements of plasma reactive oxygen metabolites (ROM) and biologic antioxidant potential (BAP) were performed to evaluate plasma oxidative status. The patients were assigned into 2 groups as low and high plasma ROM level using a cut-off value which was median of plasma ROM level at baseline. Results. In the subjects with low plasma ROM level at baseline, changes in mean CAL were positively correlated with changes in plasma ROM levels, bleeding on probing, and plaque control record, but not with PPD. In the subjects with high plasma ROM at baseline, changes in CAL were significantly associated with only PPD at baseline. On the other hands there were no significant associations between changes in CAL and those in plasma BAP levels. Conclusions. When plasma ROM level in periodontitis patients was low, increases in plasma ROM level were associated with those in CAL during the maintenance phase of treatment.
\end{abstract}

\section{Introduction}

Periodontitis is one of the most widespread chronic diseases and is characterized by gingival bleeding, periodontal pocket formation, destruction of connective tissue attachment, and alveolar bone resorption. The primary etiological agent for periodontitis is dental plaque bacteria $[1,2]$. However, it is also recognized that the majority of periodontal tissue destruction is caused by abnormal host responses to these microorganisms and their products $[3,4]$.

Reactive oxygen species (ROS) is a collective term that includes superoxide, hydroxyl, and nitric oxide radical species, as well as nonradical derivatives of oxygen. Production of ROS is a normal part of cellular metabolism.
However, overproduction of ROS disrupts the tissue oxidative/antioxidative balance, which induces oxidative injury by damaging DNA, lipids, and proteins [5]. Clinical studies have noted that periodontitis patients have elevated blood levels of total oxidative status [6,7], lipid peroxidation [6], and protein oxidation [8] compared to periodontally healthy subjects. In addition, it has been shown that periodontal treatment decreases plasma oxidized lowdensity lipoprotein levels [9] and serum total oxidative status [10] in chronic periodontitis patients. Although it is still unclear whether oxidative status is the cause or result of periodontitis [10-12], it has been suggested that systemic increases in oxidative status can affect periodontitis progression. 
Increases in oxidative status are involved in the progression of various diseases, including cardiovascular diseases [13], diabetes mellitus [14], liver diseases [15], and periodontitis $[11,16]$. Furthermore, reviews have suggested that oxidative status acts as a potential common link that explains the relationship between periodontal and systemic conditions $[17,18]$. Thus, measuring systemic oxidative status in periodontitis patients may be useful for evaluating the effects of systemic conditions on periodontal health.

The maintenance phase of periodontal treatment is important for maintaining a stable periodontal condition after initial preparation therapy, periodontal surgery, or therapy for recovering oral function. Unfortunately, periodontitis continues to progress in some patients, even during the maintenance phase of treatment. Since there is a positive correlation between changes in periodontal and systemic health [19-21], progressive periodontitis during the maintenance phase may also be associated with alterations in systemic health, including systemic increases in oxidative status. However, the longitudinal relationship between periodontal conditions and systemic oxidative status during the maintenance phase remains unclear.

Recently, a method for measuring reactive oxygen metabolites (ROM) has been found to be useful for the evaluation of oxidative status in the circulation [22-24]. This test measures the level of generic peroxide present in the plasma, which in turn reflects the level of free radicals from which they were formed. A close correlation between ROM levels and hydrogen peroxide concentrations in serum samples $(r=0.94, P<0.001)$ supports the validity of measuring ROM to evaluate oxidative status [25]. Furthermore, as ROM measurement can be performed within 10 minutes, the feasibility of clinical monitoring of plasma ROM levels in periodontitis patients is high.

In the present study, we hypothesized that systemic increases in oxidative status might contribute to periodontitis progression during the maintenance phase of periodontal treatment. The purpose of this cohort study was therefore to investigate the longitudinal relationship between plasma ROM levels and periodontitis progression during the maintenance phase of therapy. To avoid the effects of the other systemic conditions on periodontitis progression, we excluded smokers and the subjects who had diabetes mellitus, liver diseases, cancer, dyslipidemia, lung diseases, and renal diseases. In addition, since aging [26] can affect oxidative status during a follow-up period, we examined the correlation between changes in periodontal condition and the continuous variables, including age. Furthermore, plasma levels of biologic antioxidant potential (BAP) were also measured to evaluate the corresponding antioxidative status $[22,27,28]$. This is a photometric test that measures the plasma biological antioxidant potential as the capacity of the plasma sample to reduce iron from ferric $\left(\mathrm{Fe}^{3+}\right)$ to ferrous form $\left(\mathrm{Fe}^{2+}\right)$.

\section{Materials and Methods}

2.1. Study Population. The patients participating in the present study were the same patient population (81 chronic periodontitis patients, 62 women and 19 men) characterized in our previous cross-sectional study [22] and were longitudinally monitored at the Department of Preventive Dentistry, Okayama University Hospital, from 2008 to 2013. All subjects received comprehensive dental care that included nonsurgical periodontal therapy consisting of oral examination, oral hygiene instructions, supra/subgingival debridement and scaling, and root-planning of all pockets $(\geq 4 \mathrm{~mm})$ every 3-4 months. At the onset of the study period (baseline), the patients had already entered the maintenance phase of therapy for more than 1 year $(9.8 \pm 7.6$ years). Exclusion criteria were as follows: pregnancy, systemic diseases (such as diabetes mellitus, liver diseases, cancer, dyslipidemia, lung diseases, and renal diseases), previous or current smoking, users of antioxidant supplements, individuals with fewer than 15 teeth, use of anti-inflammatory medicine, and/or the presence of acute periodontal inflammation within the 3-month period prior to the start of the study period and final examinations. Due to several factors (26 moved away, 7 had medical problems, and 3 had data missing), 36 patients were lost prior to the follow-up examinations. As a result, data were analyzed for 45 subjects ( 41 women and 4 men, with a mean age of 58.8 years; response rate $55.6 \%$ ). This study was approved by the Ethics Committee of Okayama University Hospital. After obtaining informed consent, the dentists completed a detailed medical questionnaire and subjects who fulfilled the study requirements were enrolled.

2.2. Oral Examination. Probing pocket depth (PPD) and clinical attachment level (CAL) were determined at six sites (mesiobuccal, mid-buccal, distobuccal, mesiolingual, midlingual, and distolingual) on all teeth using a color-coded probe (Hu-Friedy, Chicago, IL, USA). Sites that bled upon gentle probing ( $25 \mathrm{~g}$ probing force) were recorded, and the proportion of sites with bleeding on probing (BOP) was measured in each subject. The plaque control record (PCR) was measured using erythrosine staining and was recorded with respect to their relative location to the gingival margin at four sites (mesial, distal, buccal, and lingual) around each tooth [29]. All clinical procedures were performed by four trained and calibrated dentists (Takaaki Tomofuji, Yuya Kawabata, Daisuke Ekuni and Naofumi Tamaki). In order to assess intra- and interexaminer agreement, measurements of PPD and CAL were recorded and repeated within a 2 -week interval for 8 volunteers randomly selected from the cohort of chronic periodontitis patients. Data were analyzed using the nonparametric $\kappa$ test and intraclass correlation. The $\kappa$ coefficients for intra- and interexaminer and intraclass correlation coefficients were $>0.8$.

2.3. Blood Sampling. Blood samples were obtained from the fingertip and immediately placed on ice. Following centrifugation at $3,000 \times \mathrm{g}$ for $5 \mathrm{~min}$, ROM levels were measured in 
the obtained plasma samples. Measurements of ROM levels were performed on the same day as blood sampling.

2.4. Measurement of Plasma ROM Levels. Plasma ROM levels were determined using a free radical evaluator (Diacron International, Grosseto, Italy), in accordance with previously reported procedures $[22,23]$.

The reaction utilized in this system is outlined below.

Step 1. R-OOH (generic peroxide) $+\mathrm{Fe}^{2+} \rightarrow \mathrm{R}-\mathrm{O}^{*}$ (the alkoxyl radical of a generic peroxide) $+\mathrm{Fe}^{3+}+\mathrm{OH}^{-}$or $\mathrm{R}-\mathrm{OOH}+\mathrm{Fe}^{3+} \rightarrow \mathrm{R}-\mathrm{OO}^{*}$ (the peroxyl radical of a generic peroxide $)+\mathrm{Fe}^{2+}+\mathrm{H}^{+}$or $\mathrm{R}-\mathrm{O}^{*}+[\mathrm{Fe}=\mathrm{O}]^{2+}+\mathrm{H}^{+}$.

Step 2. $\mathrm{R}-\mathrm{O}^{*}$ or $\mathrm{R}-\mathrm{OO}^{*}+\mathrm{A}-\mathrm{NH}_{2}$ (N,N-diethyl-paraphenylendiamine [chromogenic substrate] $) \rightarrow \mathrm{R}^{-} \mathrm{O}^{-}$or $\mathrm{R}-\mathrm{OO}^{-}+\left[\mathrm{A}-\mathrm{NH}_{2}^{*}\right]^{+}$(colored).

$\left[\mathrm{A}-\mathrm{NH}_{2}^{*}\right]^{+}$is the colored radical cation of the chromogenic substrate that is spectrophotometrically detectable at $505 \mathrm{~nm}$.

The results of measurements are expressed in arbitrary units (CARR U; derived from the name of the chemist [Carratelli] who invented the test), with 1 CARR U being equivalent to $0.08 \mathrm{mg} / \mathrm{dL}$ hydrogen peroxide.

The reliability of plasma ROM level determinations was confirmed by assessing plasma samples extracted three times every 4 hours from the fingertips $(n=18)$, with the intraclass correlation coefficient for an individual of 0.751 . The same samples were also measured on three consecutive days for estimation of between-day reproducibility, and the coefficients of variation were $<3 \%$.

2.5. Measurement of Plasma BAP Levels. The plasma levels of $\mathrm{BAP}$ were also measured using a free radical electric evaluator (Diacron International, Grosseto, Italy), according to published analysis procedures $[22,27,28]$. A $10 \mu \mathrm{L}$ plasma sample is added to a solution of ferric chloride and thiocyanate derivate, and the intensity of any resulting decolourization is proportional to the ability of plasma to reduce ferric ions [30]. The results of the BAP test are expressed as $\mu \mathrm{mol} / \mathrm{L}$ of reduced iron. The intra-assay and the interassay coefficients of variation for this assay were $<5 \%$.

2.6. Statistical Analysis. We calculated mean PPD and mean CAL in each subject and used it for the following analysis. A paired $t$-test was used to compare the variables at baseline and 5-year follow-up. We divided the subjects with low or high oxidative status groups at baseline as follows: low (plasma ROM level $\leq 380$ CARR U) or high (plasma ROM level > 380 CARR U). This cut-off value for the oxidative status was based on the median of the present results. A Fisher's exact test and an unpaired $t$-test were used to compare the variables at baseline of the subjects with low and high oxidative status.

Pearson's correlation coefficients were calculated to determine the correlation between changes in CAL and the other continuous variables (age, number of teeth present, PPD, CAL, BOP, PCR, ROM, and BAP) at baseline and the differences in the variables between baseline and the 5-year follow-up. Multiple linear regression analyses for all subjects and subjects with low or high oxidative status at baseline were also performed to evaluate the relationships between changes in CAL and the other variables. In order to control for confounding variables, the final model included variables that had a $P<0.2$ in the bivariate analyses. We performed variance inflation factor analysis to detect and prevent multicollinearity between independent variables. Values of $P<0.05$ were considered to represent statistically significant differences.

\section{Results}

Table 1 presents the characteristics of the study population. The proportion of female patients was higher than males. According to the paired $t$-test analyses, there were significant differences between baseline and 5-year follow-up in number of teeth present, CAL, teeth with CAL $\geq 7 \mathrm{~mm}, \mathrm{BOP}, \mathrm{PCR}$, and $\operatorname{BAP}(P<0.05)$. There were no significant differences in PPD and ROM (CARR U).

Comparisons of the subjects with low and high oxidative status at baseline are shown in Table 2. Unpaired $t$-test analysis indicated that the number of teeth present and CAL were significantly different in the two groups $(P<0.05)$.

Results of the Pearson's correlation coefficients between the changes in CAL and the other parameters are shown in Tables 3, 4, and 5. In the subjects with low oxidative status at baseline, the changes in CAL were significantly associated with changes in PPD $(P<0.05)$, BOP $(P<0.05)$, PCR $(P<$ $0.05)$, and $\operatorname{ROM}(P<0.05)$ (Table 4$)$. In the all subjects and the subjects with high oxidative status at baseline, the changes in CAL were associated with PPD at baseline $(P<0.05)$ and the overall change in PPD $(P<0.05)$ (Tables 3 and 5$)$.

The results of the multiple linear regression analyses are shown in Tables 6,7 , and 8 . In the all subjects, changes in CAL were negatively correlated with PPD at baseline $(P<$ $0.05)$ and positively correlated with PCR at baseline $(P<$ 0.05) (Table 6). In the subjects with low oxidative status at baseline, changes in CAL were positively correlated with changes in BOP, PCR, and ROM $(P<0.05)$ (Table 7). In the subjects with high oxidative status at baseline, changes in CAL were negatively correlated with PPD at baseline $(P<$ 0.05) (Table 8).

\section{Discussion}

To the best of our knowledge, this is the first epidemiological study to assess the longitudinal relationship between plasma ROM levels and periodontal progression during the maintenance phase of periodontal treatment. In this study, multiple linear regression analyses showed that changes in CAL are positively correlated with changes in plasma ROM levels after adjusting for changes in BOP and PCR in the subjects with low plasma ROM levels ( $\leq 380 \mathrm{CARR} U$ ) at baseline. ROM is an indicator of the oxidative status in the circulation [22-24]. When periodontitis patients exhibit low oxidative status, it is feasible that the elevation of oxidative status in 
TABLE 1: Characteristics of the study population at baseline and 5-year follow-up.

\begin{tabular}{|c|c|c|c|}
\hline Variables & $\begin{array}{c}\text { Baseline }(N=45) \\
N(\%) \text { or } \\
\text { Mean } \pm \text { SD } \\
\end{array}$ & $\begin{array}{c}\text { Follow-up }(N=45) \\
N(\%) \text { or } \\
\text { Mean } \pm \text { SD }\end{array}$ & $P$ value ${ }^{*}$ \\
\hline Gender & & & - \\
\hline Male & $4(8.9)$ & & \\
\hline Female & $41(91.1)$ & & \\
\hline Age (years) & $58.8 \pm 10.1$ & $64.6 \pm 10.2$ & - \\
\hline Teeth present $(n)$ & $25.0 \pm 4.4$ & $24.1 \pm 4.9$ & $<0.001$ \\
\hline \multicolumn{4}{|l|}{ Periodontal parameters } \\
\hline $\mathrm{PPD}(\mathrm{mm})$ & $1.8 \pm 0.3$ & $1.9 \pm 0.3$ & 0.107 \\
\hline CAL $(\mathrm{mm})$ & $2.6 \pm 1.1$ & $2.8 \pm 1.1$ & 0.001 \\
\hline Teeth with CAL $\geq 7 \mathrm{~mm}(n)$ & $1.2 \pm 2.9$ & $1.7 \pm 3.6$ & $<0.001$ \\
\hline Sites with BOP (\%) & $4.5 \pm 4.1$ & $7.7 \pm 7.6$ & 0.012 \\
\hline PCR (\%) & $16.0 \pm 13.4$ & $21.3 \pm 17.8$ & 0.044 \\
\hline \multicolumn{4}{|l|}{ Plasma parameters } \\
\hline ROM (CARR U) & $390.6 \pm 61.0$ & $389.4 \pm 63.8$ & 0.913 \\
\hline $\mathrm{BAP}(\mu \mathrm{mol} / \mathrm{L})$ & $2380.6 \pm 244.8$ & $1784.1 \pm 501.5$ & $<0.001$ \\
\hline
\end{tabular}

* Paired $t$-test.

$N$ : number; SD: standard deviation; PPD: probing pocket depth; CAL: clinical attachment level; BOP: bleeding on probing; PCR: plaque control record; ROM: reactive oxygen metabolites; CARR U: Carratelli units; and BAP: biologic antioxidant potential.

TABLE 2: Comparisons of each parameter at baseline between the subjects with low oxidative status ( $\leq 380 \mathrm{CARR} U$ ) and high oxidative status (>380 CARR U).

\begin{tabular}{|c|c|c|c|}
\hline \multirow{2}{*}{ Variables } & \multicolumn{2}{|c|}{ Plasma ROM Level (CARR U) } & \multirow{2}{*}{$P$ value } \\
\hline & $\begin{array}{l}\text { Low oxidative status } \\
\quad(N=23) \\
N(\%) \text { or Mean } \pm \text { SD }\end{array}$ & $\begin{array}{l}\text { High oxidative status } \\
\qquad(N=22) \\
N(\%) \text { or Mean } \pm \text { SD }\end{array}$ & \\
\hline Gender & & & $0.608^{*}$ \\
\hline Male & $3(13.0)$ & $1(4.5)$ & \\
\hline Female & $20(87.0)$ & $21(95.5)$ & \\
\hline Age (years) & $57.6 \pm 9.4$ & $60.1 \pm 11.0$ & $0.418^{\dagger}$ \\
\hline Teeth present $(n)$ & $26.7 \pm 3.0$ & $23.2 \pm 5.0$ & $0.009^{\dagger}$ \\
\hline $\operatorname{PPD}(\mathrm{mm})$ & $1.8 \pm 0.3$ & $1.8 \pm 0.4$ & $0.959^{\dagger}$ \\
\hline $\mathrm{CAL}(\mathrm{mm})$ & $2.1 \pm 0.4$ & $3.0 \pm 1.4$ & $0.010^{\dagger}$ \\
\hline Teeth with CAL $\geq 7 \mathrm{~mm}(n)$ & $0.4 \pm 0.6$ & $2.1 \pm 4.0$ & $0.053^{\dagger}$ \\
\hline $\mathrm{BOP}(\%)$ & $4.9 \pm 4.3$ & $4.2 \pm 3.9$ & $0.578^{\dagger}$ \\
\hline $\operatorname{PCR}(\%)$ & $17.0 \pm 13.9$ & $15.1 \pm 13.2$ & $0.640^{\dagger}$ \\
\hline $\mathrm{BAP}(\mu \mathrm{mol} / \mathrm{L})$ & $2350.9 \pm 230.0$ & $2411.6 \pm 261.0$ & $0.412^{\dagger}$ \\
\hline
\end{tabular}

${ }^{*}$ Fisher's exact test. ${ }^{\dagger}$ Unpaired $t$-test.

$N$ : number; SD: standard deviation; PPD: probing pocket depth; CAL: clinical attachment level; BOP: bleeding on probing; PCR: plaque control record; ROM: reactive oxygen metabolites; CARR U: Carratelli units; and BAP: biologic antioxidant potential.

the circulation could contribute to periodontitis progression during the maintenance phase.

At baseline, all subjects in this study had already received a supportive periodontal care program for more than 1 year. The low percentage of sites with BOP and dental plaque at baseline and final examinations reflected the characteristics of a well-maintained periodontal patient population. In this study, the results show that periodontitis progression was correlated with plasma ROM levels, even when low percentages of BOP and PCR were present. These suggest that periodontitis progression in the present population was associated with systemic risk factors for periodontitis. Animal studies have demonstrated that increases in serum ROM can lead to the progression of periodontitis [31, 32]. In addition, recent studies report that dietary habits influence serum ROM levels [33]. Although further studies are needed, therapeutic approaches exploiting alterations in dietary habits may offer clinical benefits to the prevention of 
TABLE 3: Pearson's correlation coefficients between change of CAL and the independent variables in the all subjects $(N=45)$.

\begin{tabular}{|c|c|c|}
\hline Independent variables & $r^{*}$ between $\Delta \mathrm{CAL}$ and the independent variables & $P$ value \\
\hline \multicolumn{3}{|l|}{ Variables at baseline } \\
\hline Age (years) & -0.058 & 0.707 \\
\hline Teeth present $(n)$ & 0.048 & 0.754 \\
\hline $\mathrm{PPD}(\mathrm{mm})$ at baseline & -0.427 & 0.003 \\
\hline CAL $(\mathrm{mm})$ at baseline & -0.138 & 0.367 \\
\hline $\mathrm{BOP}(\%)$ at baseline & -0.048 & 0.752 \\
\hline PCR (\%) at baseline & 0.240 & 0.112 \\
\hline ROM (CARR U) at baseline & 0.134 & 0.381 \\
\hline $\mathrm{BAP}(\mu \mathrm{mol} / \mathrm{L})$ at baseline & 0.004 & 0.982 \\
\hline \multicolumn{3}{|l|}{ Differences of variables } \\
\hline$\Delta$ Teeth present $(n)$ & -0.288 & 0.055 \\
\hline$\triangle \mathrm{PPD}(\mathrm{mm})$ & 0.583 & $<0.001$ \\
\hline$\triangle \mathrm{BOP}(\%)$ & 0.021 & 0.891 \\
\hline$\triangle \mathrm{PCR}(\%)$ & 0.115 & 0.450 \\
\hline$\triangle \mathrm{ROM}(\mathrm{CARR} \mathrm{U})$ & 0.078 & 0.609 \\
\hline$\Delta \mathrm{BAP}(\mu \mathrm{mol} / \mathrm{L})$ & -0.085 & 0.599 \\
\hline
\end{tabular}

$\Delta$ represents differences (value at follow-up minus value at baseline).

${ }^{*}$ Pearson's correlation coefficients.

$N$ : number; SD: standard deviation; PPD: probing pocket depth; CAL: clinical attachment level; BOP: bleeding on probing; PCR: plaque control record; ROM: reactive oxygen metabolites; CARR U: Carratelli units; and BAP: biologic antioxidant potential.

TABLE 4: Pearson's correlation coefficients between change of CAL and the independent variables in the subjects with low oxidative status $(\leq 380$ CARR $U)(N=23)$.

\begin{tabular}{|c|c|c|}
\hline Independent variables & $r^{*}$ between $\Delta \mathrm{CAL}$ and the independent variables & $P$ value \\
\hline \multicolumn{3}{|l|}{ Variables at baseline } \\
\hline Age (years) & -0.082 & 0.711 \\
\hline Teeth present $(n)$ & 0.012 & 0.956 \\
\hline PPD $(\mathrm{mm})$ at baseline & -0.268 & 0.217 \\
\hline CAL $(\mathrm{mm})$ at baseline & -0.183 & 0.402 \\
\hline $\mathrm{BOP}(\%)$ at baseline & -0.219 & 0.315 \\
\hline PCR (\%) at baseline & 0.305 & 0.157 \\
\hline ROM (CARR U) at baseline & 0.062 & 0.778 \\
\hline $\operatorname{BAP}(\mu \mathrm{mol} / \mathrm{L})$ at baseline & 0.038 & 0.863 \\
\hline \multicolumn{3}{|l|}{ Differences of variables } \\
\hline$\Delta$ Teeth present $(n)$ & -0.228 & 0.296 \\
\hline$\triangle \mathrm{PPD}(\mathrm{mm})$ & 0.660 & 0.001 \\
\hline$\triangle \mathrm{BOP}(\%)$ & 0.563 & 0.005 \\
\hline$\triangle \mathrm{PCR}(\%)$ & 0.487 & 0.018 \\
\hline$\triangle \mathrm{ROM}(\mathrm{CARR} \mathrm{U})$ & 0.443 & 0.034 \\
\hline$\Delta \mathrm{BAP}(\mu \mathrm{mol} / \mathrm{L})$ & 0.240 & 0.322 \\
\hline
\end{tabular}

$\Delta$ represents differences (value at follow-up minus value at baseline).

${ }^{*}$ Pearson's correlation coefficients.

N: number; SD: standard deviation; PPD: probing pocket depth; CAL: clinical attachment level; BOP: bleeding on probing; PCR: plaque control record; ROM: reactive oxygen metabolites; CARR U: Carratelli units; and BAP: biologic antioxidant potential.

periodontitis progression in patients during the maintenance phase of periodontal treatment.

Clinical studies have investigated the effects of antioxidant supplementation in periodontitis patients. For instance, it has been reported that supplementation of $8 \mathrm{mg} /$ day lycopene produced a significantreduction in gingival bleed- ing when compared to controls [34]. A double-blind randomized controlled trial also showed that daily supplementation with a juice powder containing an antioxidant phytonutrient strengthened the effects of periodontal treatment on the reduction of PPD, relative to placebo [35]. These observations are consistent with the present results supporting the notion 
TABLE 5: Pearson's correlation coefficients between change of CAL and the independent variables in the subjects with high oxidative status $(>380$ CARR U) $(N=22)$.

\begin{tabular}{|c|c|c|}
\hline Independent variables & $r^{*}$ between $\Delta$ CAL and the independent variables & $P$ value \\
\hline \multicolumn{3}{|l|}{ Variables at baseline } \\
\hline Age (years) & -0.075 & 0.742 \\
\hline Teeth present $(n)$ & 0.152 & 0.500 \\
\hline PPD $(\mathrm{mm})$ at baseline & -0.531 & 0.011 \\
\hline CAL $(\mathrm{mm})$ at baseline & -0.228 & 0.308 \\
\hline BOP (\%) at baseline & 0.092 & 0.683 \\
\hline PCR (\%) at baseline & 0.233 & 0.298 \\
\hline ROM (CARR U) at baseline & 0.032 & 0.887 \\
\hline $\mathrm{BAP}(\mu \mathrm{mol} / \mathrm{L})$ at baseline & -0.044 & 0.845 \\
\hline \multicolumn{3}{|l|}{ Differences of variables } \\
\hline$\Delta$ Teeth present $(n)$ & -0.352 & 0.108 \\
\hline$\triangle \mathrm{PPD}(\mathrm{mm})$ & 0.576 & 0.005 \\
\hline$\triangle \mathrm{BOP}(\%)$ & -0.235 & 0.293 \\
\hline$\triangle \mathrm{PCR}(\%)$ & -0.358 & 0.102 \\
\hline$\triangle \mathrm{ROM}(\mathrm{CARR} \mathrm{U})$ & -0.075 & 0.741 \\
\hline$\Delta \mathrm{BAP}(\mu \mathrm{mol} / \mathrm{L})$ & -0.256 & 0.249 \\
\hline
\end{tabular}

$\Delta$ represents differences (value at follow-up minus value at baseline).

${ }^{*}$ Pearson's correlation coefficients.

$N$ : number; SD: standard deviation; PPD: probing pocket depth; CAL: clinical attachment level; BOP: bleeding on probing; PCR: plaque control record; ROM: reactive oxygen metabolites; CARR U: Carratelli units; and BAP: biologic antioxidant potential.

TABLE 6: Multiple linear regression analysis with change of CAL as the dependent variable in the all subjects $(N=45)$.

\begin{tabular}{lccc}
\hline Variables & $B(95 \% \mathrm{CI})$ & $\beta$ & $P$ value \\
\hline Intercept & $1.068(0.470,1.666)$ & - & 0.001 \\
PPD $(\mathrm{mm})$ at baseline & $-0.534(-0.853,-0.216)$ & -0.451 & 0.002 \\
PCR $(\%)$ at baseline & $0.008(0.0003,0.016)$ & 0.262 & 0.042 \\
\hline
\end{tabular}

We used backward elimination method with a $P>0.1$ threshold for exclusion to construct the model. The candidate variables for the model were $\Delta$ Teeth present, PPD at baseline, and PCR at baseline. The F-statistic was $7.374(P$ value was 0.002$)$, the $R$-square was 0.260 , and the adjusted $R$-square of the model was 0.225 . The variance inflation factor was $<5$ and condition index was $<15$.

$\Delta$ represents differences (value at follow-up minus value at baseline).

$N$ : number; CI: confidence interval; $B$ : unstandardized regression coefficient; $\beta$ : standardized coefficient; PPD: probing pocket depth; CAL: clinical attachment level; PCR: plaque control record.

that systemic approaches to ROS reduction may be clinically effective in suppressing periodontitis progression.

In the subjects with high oxidative status ( $>380$ CARR $\mathrm{U})$ at baseline, there was no correlation between changes in CAL and changes in ROM. At baseline, periodontitis patients with high oxidative status had greater CAL than those with low oxidative status. The lack of correlation between changes in periodontal condition and ROM in periodontitis patients with high oxidative status might be due to the severity of periodontal disease already present at baseline.

Several studies have investigated the relationship between blood ROM levels and chronic diseases. For instance, clinical studies have demonstrated that high ROM levels could be a predictor of cardiovascular disease progression $[36,37]$. It is also known that high ROM levels are an independent predictor of obesity (odds ratio: $2.5 ; P<0.001$ ) in women [38]. These observations show that increases in plasma ROM may contribute to the progression of chronic diseases. In this study, increases in plasma ROM levels were associated with changes in CAL in subjects with low plasma ROM levels at baseline. Although further studies are needed, it is possible that monitoring plasma ROM levels is a useful method for monitoring the effects of systemic conditions on periodontal health in periodontitis patients.

In our previous cross-sectional study [22], plasma ROM levels were positively associated with CAL in chronic periodontitis patients. We also found that nonsurgical periodontal treatment improved both periodontal inflammation and plasma ROM in chronic periodontitis patients [23]. Furthermore, the present findings indicated that the increased plasma ROM levels might contribute to changes in periodontal conditions. These results suggest that there is a close relationship between periodontal conditions and systemic oxidative status.

In this study, alterations in CAL were negatively correlated with PPD at baseline in subjects with high oxidative status. Recent research has suggested that baseline PPD is associated with subsequent alveolar bone density and height loss in postmenopausal osteopenic females undergoing periodontal maintenance [39]. However, it have been indicated that sites with deep PPD showed more attachment level gain after 3 months of scaling and root planning than those with shallow PPD [40]. In addition, it has been also reported that attachment level gain after 3 months of curettage of periodontal pockets was greater in deep PPD than in shallow PPD [41]. Since deep PPD may be affected by periodontal maintenance therapy to a greater extent than shallow PPD, 
TABLE 7: Multiple linear regression analysis with change of CAL as the dependent variable in the subjects with low oxidative status ( $\leq 380$ CARR U) $(N=23)$.

\begin{tabular}{lccc}
\hline Variables & $B(95 \%$ CI $)$ & $\beta$ & $P$ value \\
\hline Intercept & $0.044(-0.060,0.148)$ & - & 0.391 \\
$\Delta$ BOP (\%) & $0.013(0.0003,0.025)$ & 0.349 & 0.045 \\
$\Delta$ PCR (\%) & $0.006(0.001,0.010)$ & 0.409 & 0.019 \\
$\Delta$ ROM (CARR U) & $0.002(0.0004,0.003)$ & 0.416 & 0.014 \\
\hline
\end{tabular}

We used backward elimination method with a $P>0.1$ threshold for exclusion to construct the Model. The candidate variables for the Model were $\triangle \mathrm{BOP}, \triangle \mathrm{PCR}$, and $\triangle \mathrm{ROM}$. The $F$-statistic was 8.744 ( $P$-value was 0.001$)$, the $R$-square was 0.580 , and the adjusted $R$-square of the model was 0.514 . The variance inflation factor was $<5$ and condition index was $<15$.

$\Delta$ represents differences (value at follow-up minus value at baseline).

$N$ : number, CI: confidence interval, $B$ : unstandardized regression coefficient, $\beta$ : standardized coefficient, CAL: clinical attachment level, BOP: bleeding on probing, PCR: plaque control record, CARR U: and Carratelli units.

TABLE 8: Multiple linear regression analysis with change of CAL as the dependent variable in the subjects with high oxidative status (>380 CARR U) $(N=22)$.

\begin{tabular}{lccc}
\hline Variables & $B(95 \% \mathrm{CI})$ & $\beta$ & $P$ value \\
\hline Intercept & $1.622(0.650,2.593)$ & - & 0.002 \\
PPD $(\mathrm{mm})$ at baseline & $-0.718(-1.236,-0.200)$ & -0.539 & 0.009 \\
$\Delta$ PCR $(\%)$ & $-0.011(-0.027,0.006)$ & -0.251 & 0.193 \\
\hline
\end{tabular}

We used backward elimination method with a $P>0.1$ threshold for exclusion to construct the Model. The candidate variables for the model were $\Delta$ Teeth present, PPD at baseline, and $\triangle$ PCR. The $F$-statistic was 5.012 ( $P$ value was 0.018 ), the $R$-square was 0.345 , and the adjusted $R$-square of the model was 0.276 . The variance inflation factor was $<5$ and condition index was $<15$.

$\Delta$ represents differences (value at follow-up minus value at baseline).

$N$ : number; CI: confidence interval; $B$ : unstandardized regression coefficient; $\beta$ : standardized coefficient; PPD: probing pocket depth; CAL: clinical attachment level; PCR: plaque control record; and CARR U: Carratelli units.

the correlation between changes in CAL and PPD at baseline was negative in our study.

On the other hand, plasma BAP levels were not correlated with changes in CAL in this study. This indicates that the correlation between blood total antioxidant status and periodontitis progression is modest. Several methodologies are now available to evaluate blood antioxidant status, and a previous study suggested that the $\mathrm{Fe}^{3+}$ reducing antioxidant power assay used in our study has certain drawbacks, from the viewpoint of interference and reaction kinetics, in comparison with the oxygen radical absorption capacity (ORAC) method [42]. Therefore, the failure to observe a correlation between plasma antioxidative status and change in periodontal condition could be related to the choice of the BAP test. In a potential future study, additional assays (e.g., ORAC method) may be required to elucidate the correlation between systemic antioxidant status and change in periodontal condition.

An acknowledged weakness of this study is that all subjects were recruited at the Okayama University Hospital. This may limit the ability to generalize our findings to the broader population of patients in the maintenance phase of periodontal treatment. In addition, we did not use strict measures to control factors (C-reactive protein and dental biofilms) that might influence plasma ROM and periodontal conditions. In particular, it is reported that serum ROM levels are closely associated with serum high-sensitivity C-reactive protein in a Japanese population [43]. Measurement of highsensitivity C-reactive protein would increase the validity of the presently established relationship between plasma ROM levels and periodontitis progression. In addition, the lack of annual follow-ups was also the limitation of the study because oxidative status may have not continued to simply increase or decrease.

\section{Conclusion}

In the subjects with low plasma ROM level ( $\leq 380$ CARR U) at baseline, changes in mean CAL were positively correlated with those in plasma ROM levels during the maintenance phase of periodontal treatment. On the other hand, in the subjects with high plasma ROM level ( $>380$ CARR U) at baseline, changes in mean CAL were not correlated with those in plasma ROM levels. Furthermore, there were no correlation between changes in mean CAL and those in plasma BAP levels.

\section{Conflict of Interests}

The authors have no conflict of interests to declare.

\section{Acknowledgment}

This work was supported by Grants-in-Aid for Scientific Research (nos. 20335615 and 23390480) from the Ministry of Education, Culture, Sports, Science and Technology, Tokyo, Japan. 


\section{References}

[1] P. N. Madianos, Y. A. Bobetsis, and D. F. Kinane, "Generation of inflammatory stimuli: how bacteria set up inflammatory responses in the gingiva," Journal of Clinical Periodontology, vol. 32, no. 6, pp. 57-71, 2005.

[2] A. P. V. Colombo, S. K. Boches, S. L. Cotton et al., "Comparisons of subgingival microbial profiles of refractory periodontitis, severe periodontitis, and periodontal health using the human oral microbe identification microarray," Journal of Periodontology, vol. 80, no. 9, pp. 1421-1432, 2009.

[3] R. C. Page and K. S. Kornman, "The pathogenesis of human periodontitis: an introduction," Periodontology 2000, vol. 14, pp. 9-11, 1997.

[4] M. Benakanakere and D. F. Kinane, "Innate cellular responses to the periodontal biofilm," Frontiers of oral biology, vol. 15, pp. 41-55, 2012.

[5] B. Halliwell, "Free radicals, antioxidants, and human disease: curiosity, cause, or consequence?” The Lancet, vol. 344, no. 8924, pp. 721-724, 1994.

[6] F. A. Akalin, E. Baltacioğlu, A. Alver, and E. Karabulut, "Lipid peroxidation levels and total oxidant status in serum, saliva and gingival crevicular fluid in patients with chronic periodontitis," Journal of Clinical Periodontology, vol. 34, no. 7, pp. 558-565, 2007.

[7] E. Baltacioğlu, M. A. Kehribar, P. Yuva et al., “Total oxidant status and bone resorption biomarkers in serum and gingival crevicular fluid of patients with periodontitis," Journal of Periodontology, vol. 85, no. 2, pp. 317-326, 2014.

[8] E. Baltacioğlu, F. A. Akalin, A. Alver, O. Deǧer, and E. Karabulut, "Protein carbonyl levels in serum and gingival crevicular fluid in patients with chronic periodontitis," Archives of Oral Biology, vol. 53, no. 8, pp. 716-722, 2008.

[9] N. Tamaki, T. Tomofuji, D. Ekuni, R. Yamanaka, and M. Morita, "Periodontal treatment decreases plasma oxidized LDL level and oxidative stress," Clinical Oral Investigations, vol. 15, no. 6, pp. 953-958, 2011.

[10] A. Akpinar, H. Toker, H. Ozdemir, V. Bostanci, and H. Aydin, "The effects of non-surgical periodontal therapy on oxidant and anti-oxidant status in smokers with chronic periodontitis," Archives of Oral Biology, vol. 58, pp. 717-723, 2013.

[11] I. L. C. Chapple and J. B. Matthews, "The role of reactive oxygen and antioxidant species in periodontal tissue destruction," Periodontology 2000, vol. 43, no. 1, pp. 160-232, 2007.

[12] P. Dahiya, R. Kamal, R. Gupta, R. Bhardwaj, K. Chaudhary, and S. Kaur, "Reactive oxygen species in periodontitis," Journal of Indian Society of Periodontology, vol. 17, pp. 411-416, 2013.

[13] G. Daffu, C. H. Del Pozo, K. M. O’'Shea, R. Ananthakrishnan, R. Ramasamy, and A. M. Schmidt, "Radical roles for RAGE in the pathogenesis of oxidative stress in cardiovascular diseases and beyond," International Journal of Molecular Sciences, vol. 14, pp. 19891-19910, 2013.

[14] K. Stadler, "Oxidative stress in diabetes," Advances in Experimental Medicine and Biology, vol. 771, pp. 272-287, 2012.

[15] V. Sánchez-Valle, N. C. Chávez-Tapia, M. Uribe, and N. Méndez-Sánchez, "Role of oxidative stress and molecular changes in liver fibrosis: a review," Current Medicinal Chemistry, vol. 19, pp. 4850-4860, 2012.

[16] I. L. C. Chapple, "Reactive oxygen species and antioxidants in inflammatory diseases," Journal of Clinical Periodontology, vol. 24, no. 5, pp. 287-296, 1997.
[17] E. M. Allen, J. B. Matthews, R. O'Connor, D. O'Halloran, and I. L. C. Chapple, "Periodontitis and type 2 diabetes: is oxidative stress the mechanistic link?" Scottish Medical Journal, vol. 54, no. 2, pp. 41-47, 2009.

[18] P. Bullon, J. M. Morillo, M. C. Ramirez-Tortosa, J. L. Quiles, H. N. Newman, and M. Battino, "Metabolic syndrome and periodontitis: is oxidative stress a common link?" Journal of Dental Research, vol. 88, no. 6, pp. 503-518, 2009.

[19] E. Lalla and P. N. Papapanou, "Diabetes mellitus and periodontitis: a tale of two common interrelated diseases," Nature Reviews Endocrinology, vol. 7, no. 12, pp. 738-748, 2011.

[20] G. J. Linden, A. Lyons, and F. A. Scannapieco, "Periodontal systemic associations: review of the evidence," Journal of Clinical Periodontology, vol. 40, pp. S8-S19, 2013.

[21] S. L. Shangase, G. U. Mohangi, S. Hassam-Essa, and N. H. Wood, "The association between periodontitis and systemic health: an overview," SADJ, vol. 8, pp. 10-12, 2013.

[22] N. Tamaki, T. Tomofuji, T. Maruyama et al., "Relationship between periodontal condition and plasma reactive oxygen metabolites in patients in the maintenance phase of periodontal treatment," Journal of Periodontology, vol. 79, no. 11, pp. 21362142, 2008.

[23] N. Tamaki, T. Tomofuji, D. Ekuni, R. Yamanaka, T. Yamamoto, and M. Morita, "Short-term effects of non-surgical periodontal treatment on plasma level of reactive oxygen metabolites in patients with chronic periodontitis," Journal of Periodontology, vol. 80, no. 6, pp. 901-906, 2009.

[24] K. Dohi, K. Miyamoto, K. Fukuda et al., "Status of systemic oxidative stress during therapeutic hypothermia in patients with post-cardiac arrest syndrome," Oxidative Medicine and Cellular Longevity, vol. 2013, Article ID 562429, 8 pages, 2013.

[25] I. Hayashi, Y. Morishita, K. Imai, M. Nakamura, K. Nakachi, and T. Hayashi, "High-throughput spectrophotometric assay of reactive oxygen species in serum," Mutation Research, vol. 631, no. 1, pp. 55-61, 2007.

[26] M. Andriollo-Sanchez, I. Hininger-Favier, N. Meunier et al., "Age-related oxidative stress and antioxidant parameters in middle-aged and older European subjects: the ZENITH study," European Journal of Clinical Nutrition, vol. 59, supplement 2, pp. S58-S62, 2005.

[27] K. Nakayama, H. Terawaki, M. Nakayama, M. Iwabuchi, T. Sato, and S. Ito, "Reduction of serum antioxidative capacity during hemodialysis," Clinical and Experimental Nephrology, vol. 11, no. 3, pp. 218-224, 2007.

[28] A. Pasquini, E. Luchetti, V. Marchetti, G. Cardini, and E. L. Iorio, "Analytical performances of d-ROMs test and BAP test in canine plasma. Definition of the normal range in healthy Labrador dogs," Veterinary Research Communications, vol. 32, no. 2, pp. 137-143, 2008.

[29] T. J. O’Leary, R. B. Drake, and J. E. Naylor, “The plaque control record," Journal of Periodontology, vol. 43, no. 1, p. 38, 1972.

[30] I. F. F. Benzie and J. J. Strain, "The ferric reducing ability of plasma (FRAP) as a measure of "antioxidant power": the FRAP assay," Analytical Biochemistry, vol. 239, no. 1, pp. 70-76, 1996.

[31] K. Irie, T. Tomofuji, N. Tamaki et al., "Effects of ethanol consumption on periodontal inflammation in rats," Journal of Dental Research, vol. 87, no. 5, pp. 456-460, 2008.

[32] T. Tomofuji, T. Yamamoto, N. Tamaki et al., "Effects of obesity on gingival oxidative stress in a rat model," Journal of Periodontology, vol. 80, no. 8, pp. 1324-1329, 2009. 
[33] F. Komatsu, Y. Kagawa, T. Kawabata, Y. Kaneko, and K. Ishiguro, "Relationship of dietary habits and obesity to oxidative stress in Palauan people: compared with Japanese and Mongolian people," Current Aging Science, vol. 2, no. 3, pp. 214-222, 2009.

[34] R. V. Chandra, M. L. V. Prabhuji, D. A. Roopa, S. Ravirajan, and H. C. Kishore, "Efficacy of lycopene in the treatment of gingivitis: a randomised, placebo-controlled clinical trial," Oral Health \& Preventive Dentistry, vol. 5, no. 4, pp. 327-336, 2007.

[35] I. L. C. Chapple, M. R. Milward, N. Ling-Mountford et al., "Adjunctive daily supplementation with encapsulated fruit, vegetable and berry juice powder concentrates and clinical periodontal outcomes: a double-blind RCT," Journal of Clinical Periodontology, vol. 39, no. 1, pp. 62-72, 2012.

[36] K. Kotani, H. Koibuchi, M. Miyamoto, T. Yamada, and N. Taniguchi, "Relationship between reactive oxygen metabolites and carotid intima-media thickness in subjects with hypercholesterolemia," Medical Principles and Practice, vol. 19, no. 6, pp. 496-498, 2010.

[37] C. Vassalle, S. Bianchi, D. Battaglia, P. Landi, F. Bianchi, and C. Carpeggiani, "Elevated levels of oxidative stress as a prognostic predictor of major adverse cardiovascular events in patients with coronary artery disease," Journal of Atherosclerosis and Thrombosis, vol. 19, pp. 712-717, 2012.

[38] C. Vassalle, L. Vigna, S. Bianchi et al., "A biomarker of oxidative stress as a nontraditional risk factor in obese subjects," Biomarkers in Medicine, vol. 7, pp. 633-639, 2013.

[39] J. B. Payne, P. V. Nummikoski, D. M. Thompson, L. M. Golub, and J. A. Stoner, "The association between clinical and radiographic periodontitis measurements during periodontal maintenance," Journal of Periodontology, vol. 84, pp. 1382-1390, 2013.

[40] A. D. Haffajee, M. A. Cugini, S. Dibart, C. Smith, R. L. Kent Jr., and S. S. Socransky, "Clinical and microbiological features of subjects with adult periodontitis who responded poorly to scaling and root planing," Journal of Clinical Periodontology, vol. 24, no. 10, pp. 767-776, 1997.

[41] T. Kocher, J. König, P. Hansen, and A. Rühling, "Subgingival polishing compared to scaling with steel curettes: a clinical pilot study," Journal of Clinical Periodontology, vol. 28, no. 2, pp. 194199, 2001.

[42] B. Ou, D. Huang, M. Hampsch-Woodill, J. A. Flanagan, and E. K. Deemer, "Analysis of antioxidant activities of common vegetables employing oxygen radical absorbance capacity (ORAC) and ferric reducing antioxidant power (FRAP) assays: a comparative study," Journal of Agricultural and Food Chemistry, vol. 50, no. 11, pp. 3122-3128, 2002.

[43] H. Hirose, H. Kawabe, N. Komiya, and I. Saito, "Relations between serum reactive oxygen metabolites (ROMs) and various inflammatory and metabolic parameters in a Japanese population," Journal of Atherosclerosis and Thrombosis, vol. 16, no. 2, pp. 77-82, 2009. 


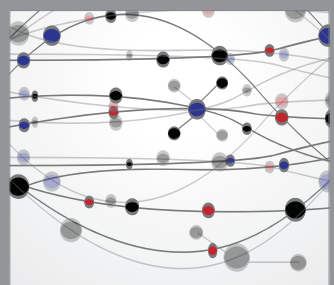

The Scientific World Journal
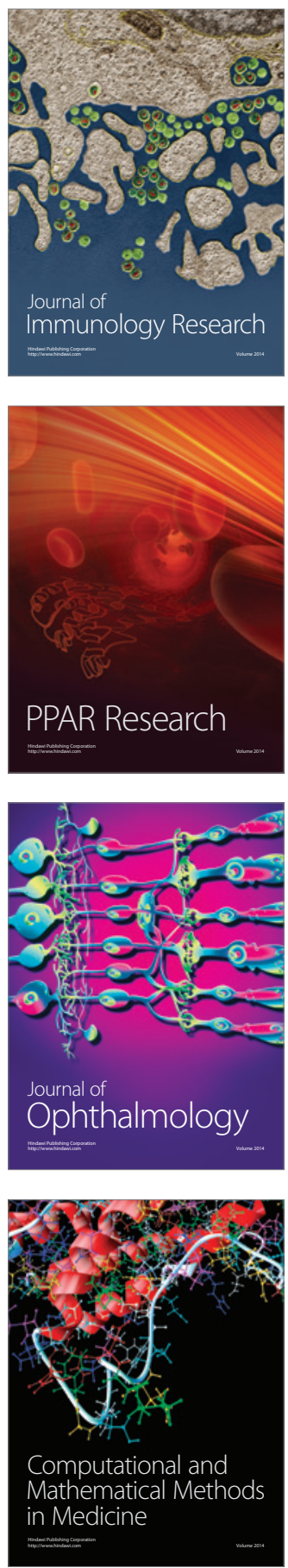

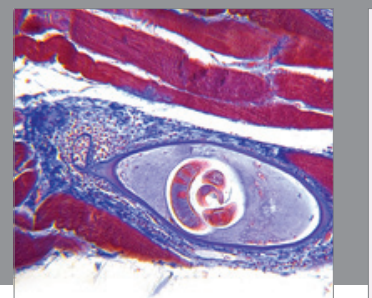

Gastroenterology

Research and Practice
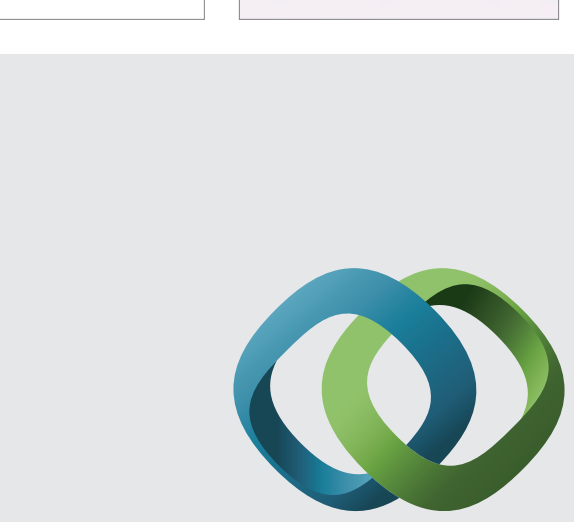

\section{Hindawi}

Submit your manuscripts at

http://www.hindawi.com
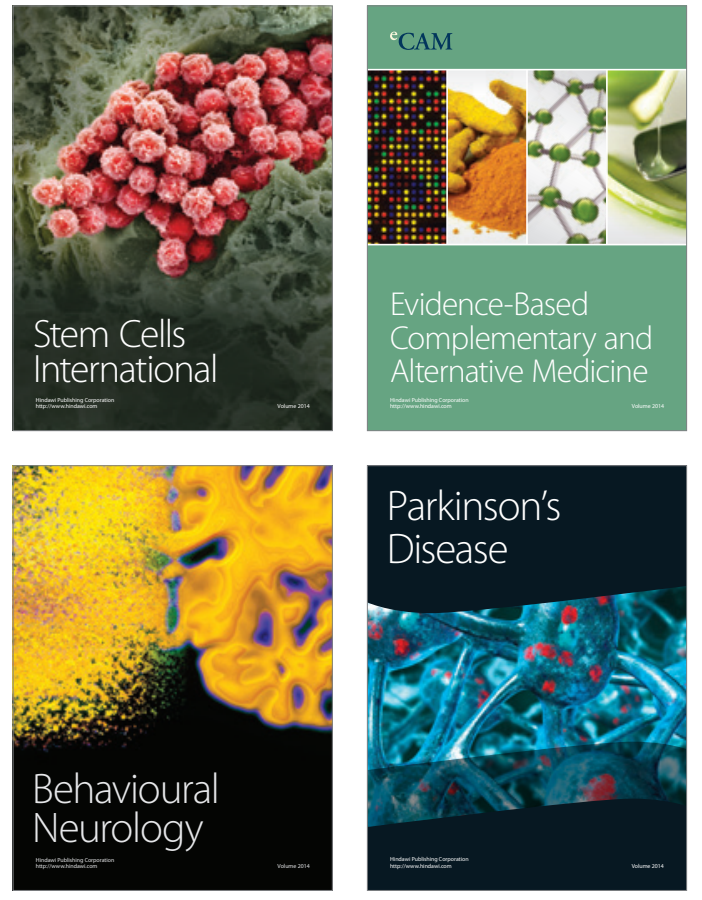
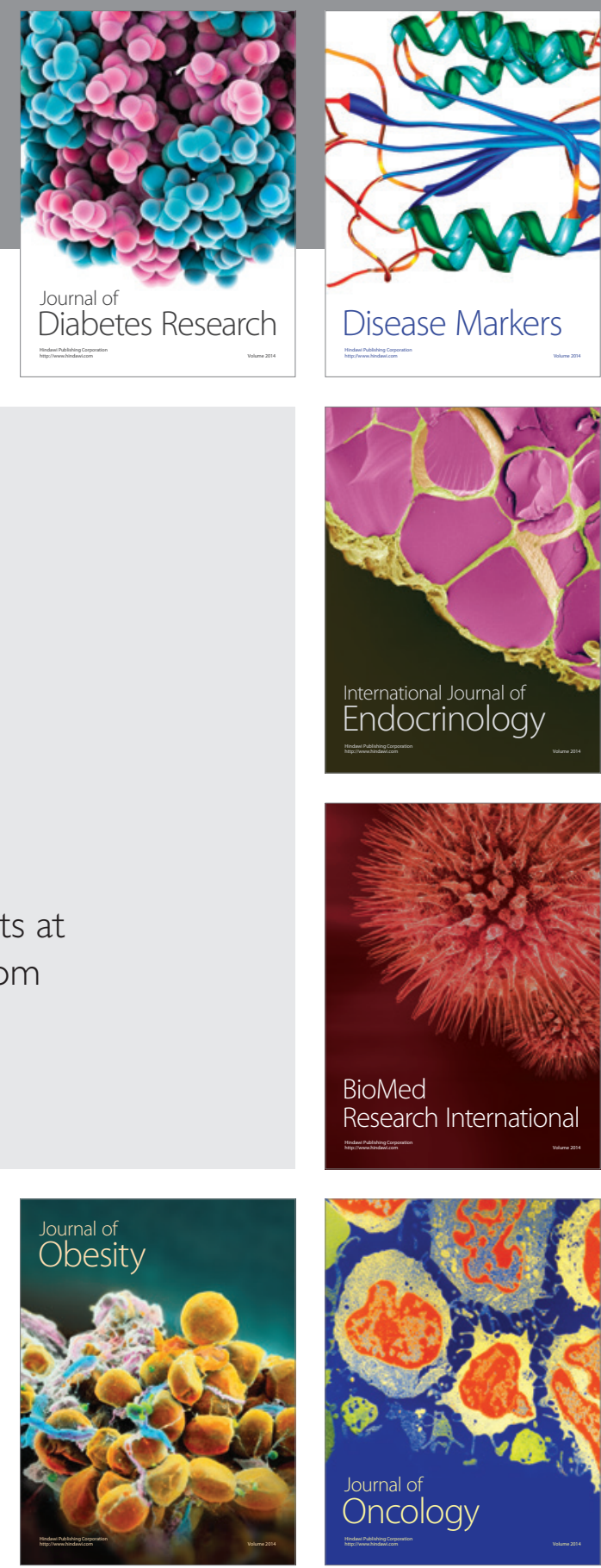

Disease Markers
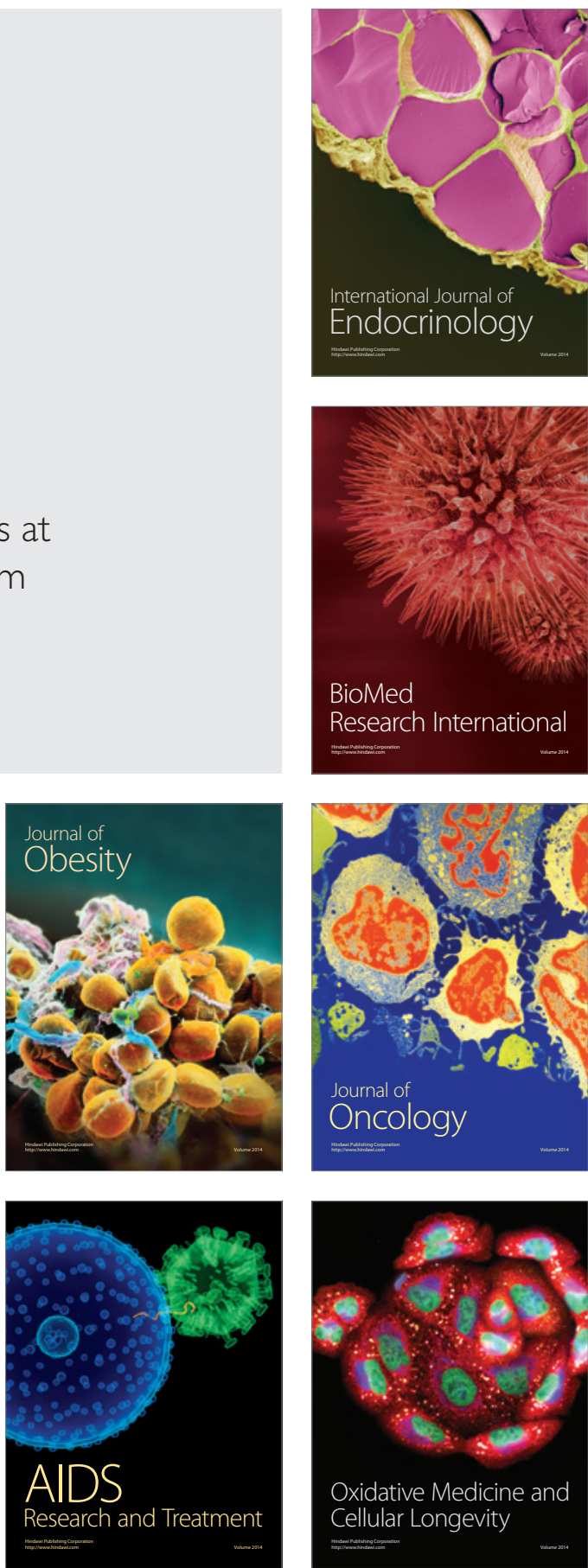